Daniela De Liso

\title{
Margherita Costa a Parigi: La selva di Diana
}

\begin{abstract}
Poetessa e virtuosa, Margherita Costa esercitò la sua poligrafia in molti generi letterari e pubblicò un considerevole numero di opere ancora oggi poco note. Il contributo vuole indagare la prima opera parigina della Costa, La Selva di Diana, pubblicata nel 1647, all'arrivo dell'autrice nella capitale francese. La Selva, che contiene testi composti nel periodo romano, dunque precedenti al soggiorno francese, avrebbe dovuto costituire il "lasciapassare" per la corte francese, che rappresentava per la poetessa un nuovo inizio. L'opera, dedicata alla principessa sabauda Cristina, sorella del re di Francia, è interamente "al femminile" e ibrida di continuo l'invenzione poetica con l'autobiografia.
\end{abstract}

The e-journal «altrelettere» is hosted at the URL: http://www.altrelettere.uzh.ch, in accordance with the Open Access Policy of the University of Zurich. Please cite this article as follows: D. DE Liso, Margherita Costa a Parigi: La selva di Diana, in «altrelettere», (2021), pp. 27-42, DOI: 10.5903/al_uzh-52.

(C) This article is licensed under a Creative Commons Attribution 2.5. Switzerland (CC BY-NC-ND 2.5). Please read the license terms on the website: http://creativecommons.org/licenses/by-nc-nd/2.5/ch/deed.en 
Margherita Costa fu una cortigiana non più «onesta» (cfr. ODORISIO 1979) e pagò lo scotto di voler essere indipendente in un mondo di uomini, in cui una donna di non nobili origini non aveva grandi chances. Nata a Roma intorno al 1600, aveva tentato di conquistare la corte di Toscana e poi era approdata nella Parigi di Mazzarino, di cui fu probabilmente amante. Fu poi a Venezia, in Toscana e di nuovo a Roma, dove concluse la sua esistenza. ${ }^{1}$ Cantante, attrice, avventuriera, innamorata di un comico e di un brigante, dopo la "scoperta" di Luisa Bergalli, che ne incluse alcuni versi nella sua raccolta di Componimenti poetici delle più illustri rimatrici d'ogni secolo (BERGALLI 1726, 149-54), nonostante la sua cospicua produzione, Margherita Costa non è amata dalla critica letteraria. Il più benevolo dei suoi studiosi datati, Dante Bianchi, tentando di giustificarne la pressoché totale mancanza di una formazione solida e, quindi, di auctores, concludeva che il merito della prolificità letteraria della Costa fosse unicamente da ascriversi all' «ingegnaccio ond'era dotata de natura» (BIANCHI 19241925, 159).

Al suo arrivo a Parigi, nel 1647, Costa aveva già scritto e pubblicato le opere più importanti e vi era probabilmente giunta come virtuosa, se il suo primo impegno pubblico fu interpretare il ruolo di Giunone nell'Orfeo di Luigi Rossi (cfr. Costa-Zalessow 2015). Con l'aiuto di Mazzarino a Parigi riuscì a pubblicare due libri di versi, La selva di Diana e La tromba di Parnaso, e uno spettacolo barocco, La Festa reale per balletto a cavallo. Si tratta delle prove di una poligrafa per vocazione, capace di spaziare tra generi e arti diverse. È, tuttavia, evidente che la versatilità della penna costiana non possa essere, in assenza di una formazione enciclopedica, niente più che un saggio della gorgiera ornatissima dell'estetica barocca. Su queste basi è legittimo chiedersi per quale ragione occorra oggi soffermarsi, riscoprendola, su questa produzione. Certamente non è una ragione il valore documentario delle opere costiane. È indubitabile, invece, che nel Seicento esista una spinosa querelle des femmes, filiazione di un'irrisolta questione femminile che nel corso del Cinquecento sembrava aver trovato nel petrarchismo e nel teatro una chiave di volta. Il ruolo di Margherita Costa e della sua produzione in questa querelle è più determinante di quanto si sia finora ritenuto, perché, di fatto, vinse la sua battaglia di affermazione da sola: non la salvarono né il chiostro, né il matrimonio, ma un ingegno, forse rozzo e per sempre acerbo, capace di far perdere la testa, non solo sentimentalmente, a principi e cardinali.

La selva di Diana è, per gli inserti autobiografici e gli spaccati di vita muliebre che ospita, la chiara dimostrazione di questo asserto. Sebbene pubblicata a Parigi, nel 1647, i suoi contenuti sono ispirati e chiaramente 
elaborati negli anni romani che avevano preceduto la permanenza francese, e ciò al di là della dedica All'Altezza reale di Madama Savoia, un omaggio dovuto a Cristina di Savoia, Madama Reale, figlia di Enrico IV e Maria de' Medici e sorella del re di Francia Luigi XIII (CosTA 1647). Nata a Parigi nel 1606, Cristina era stata data in sposa a soli tredici anni al trentunenne Vittorio Amedeo I, duca di Savoia, e spedita a Torino. Il matrimonio doveva sancire l'alleanza francosabauda in funzione antispagnola e la giovanissima Madama Reale adempì al suo ruolo di moglie, dando al marito sei figli ed ampia possibilità di successione. La morte del marito, nel 1637, rivelò poi le sue indubbie doti politiche, perché Cristina riuscì a tenere buoni i cognati, che premevano per sottrarre il trono sabaudo a suo figlio. Oltre ad essere un'abile politica, Cristina di Savoia era una donna bellissima e chiacchierata dall'austera corte torinese. Dopo la morte del marito si circondò di amanti e ammiratori e solo nell'ultima parte della sua vita ebbe una sorta di "conversione" tardiva e dispose d'essere sepolta nella semplice veste monacale. Margherita Costa, insomma, non avrebbe potuto scegliere dedicataria più sensibile alla sua arte e benevola verso uno stile di vita simile al proprio. La dedica di Costa realizza il tòpos dell'excusatio che si può ritrovare in tutte le opere di questa Autora:

\begin{abstract}
Serenissima Madama Reale,
Vogliono altri con aurati tributi prostrarsi alli piedi delle Monarchie più grandi, io all'opposito con povere, e rozze rime à piedi dell'Altezza Vostra Reale presento il piccolo parto del mio basso ingegno, e benche del Castalio regno inutil ombra io sia, supplico la Vostra Altezza Reale gradirlo, se non per la povertà di esso, per la ricchezza, che porta; mentre sotto l'aura del suo gran nome vien dato alla luce, hò voluto intitolarlo la Selva di Diana, perch'ogni invido lume che insidioso ardisse oscurarlo resti al Reggio nome di Vostra Altezza Reale un novello Atteone: con che al trono della sua protettione, con ogni riverenza maggiore a egli, ed il mio poco talento riverentemente soggiacciamo (ibidem, 4)
\end{abstract}

Non ci sono lodi sperticate all'indirizzo della dedicataria, perché esse troveranno spazio nella prima «selva» di ottave del libro, L'Alpi, una sorta di epinicio al femminile, d’interessante prospettiva.

Il Leitmotiv dell'inadeguatezza continua nel brevissimo avvertimento $A i$ Lettori, in cui, tuttavia, il discorso si fa "di genere": 


\section{Ai Lettori}

Lettore habbi riguardo nel leggere la presente mia Selva di Diana, che in petto di Donna raro s'annidano le suore d'Elicona, e come parto di sesso soggetto all'ignoranza scusa gl'errori di essa, e com'amica lingua, se non la vuoi stimar degna d'applauso, confessala di riguardo meritevole (ibidem, 5).

Scusandosi col suo Lettore per l' «ignoranza», Costa non sta facendo riferimento alla sua ignoranza soltanto, ma a quella delle donne, nei cui petti non possono annidarsi «le suore d'Elicona» non certo per difetto d'ingegno, quanto perché non a tutte loro nel Seicento è concesso studiare ed occuparsi di poesia, letteratura, arte (cfr. DE LISO 2019a). Donne di modesta origine, come lei, non possono neanche più svolgere la professione di «cortigiane oneste», come aveva fatto Veronica Franco, che per molti aspetti è la donna e l'autrice più vicina all'esperienza biografica e letteraria costiana (cfr. DE Liso 2019b). Dunque, i due brevissimi avantesti della Selva pongono le basi per un'opera interamente declinata al femminile, a partire dal titolo, sul quale occorre soffermarsi. A scorrere, infatti, l'indice, che non era incluso nell'unica edizione a stampa, ${ }^{2}$ non si evince alcun riferimento alla dea della caccia, distante dall'amore e amante solo della libertà. Il mito di Diana, divinità tutta italica e, secondo alcuni, indipendente dalla greca Artemide, di cui è considerata la filiazione, è senza dubbio connesso alla corte sabauda e, prima di essa, a quella francese di Luigi XIII, la cui sorella Maria Cristina sarà madre di quel Carlo Emanuele II che volle far costruire la Reggia di Venaria (CASTELnUOvo 2007), interamente consacrata, attraverso la mediazione di Emanuele Tesauro, al mito moralizzato di Diana:

Duchi prima, poi re cacciatori, i Savoia non a caso, affacciandosi sul palcoscenico europeo da una posizione inizialmente marginale, assumono Diana - una figura cioè dalle implicazioni ideologiche e civili immediatamente riconoscibili - come emblema della loro identità dinastica e politica: il mito della dea casta e cacciatrice evoca come meglio non si potrebbe la virtù e la pietas religiosa dei principi guerrieri che propongono il proprio Stato a baluardo della cristianità italiana contro la barbarie d'oltralpe (BARBERI SQUAROTTI 2018, X).

Insomma, nella veste di «cacciatrice ammansita» (GRISERI 1988, 195-96) da «Prudenza, Forza e Prontezza, indirizzate al fine del buon governo» (VARALLO 
2010, 141), è rappresentata nei primi schizzi decorativi della Reggia di Venaria proprio la dedicataria della Selva costiana, Maria Cristina di Savoia: ragionevolmente direi dunque che il titolo dell'opera è ulteriore omaggio a lei.

La struttura della Selva di Diana può essere così riassunta: un encomio in ottave, L'Alpi; le ottave di Artigemma alle dame laziali vendemmianti; i sonetti della settimana all'Amante; i sonetti di tema amoroso; le ottave autobiografiche dell'arrivo a Parigi e quelle della partenza da Roma. Si tratta evidentemente di un'opera pubblicata a scopo promozionale, come si evince dall'assenza di una struttura coerente, dal disordine di questa selva, nella quale unico criterio ordinatore sembra essere quello di ospitare solo versi per donne e di donna; l'universo maschile è relegato al ruolo di mero destinatario di versi di rimbrotto, dolore o denuncia. Questo carattere eminentemente 'femminile' si evince subito dalle ottave de L'Alpi, dove, dopo le prime tre strofe destinate alla topica excusatio per l'inadeguatezza della penna, Cristina di Savoia informa della sua grandezza ‘eroica' i versi:

In tè là nobiltade, e là virtute

Hanno gl'estremi lor congiunti à gara;

presso le glorie tue l'altre son mute,

e in tè di meraviglia ogn'opra è rara:

sol dà la destra tua l'alma salute

a compartir felicitade impara,

e nel tuo petto di gran merti altero

hà Febo il regno, e Pallade l'Impero.

\section{$[\ldots]$}

Cristina sovr'à l'Alpi ergi sublime

Scettro immortal'di meraviglie degno, ed a le glorie tue d'ogn'honor prime tributo di tesor porge ogni Regno:

il Barbaro Oriente non più stime dileguate grandezze: avanza il Segno in Occidente la tua prova, e sei Preggio di Pompe, e Madre di Trofei. (CosTA 1647, 8-9)

Le lodi della Regina non sono intessute in relazione agli uomini. La grandezza di Cristina non deriva dal suo essere moglie e poi madre di Re: anzi sono le doti 
di lei a rendere grandi gli uomini a lei legati. Le divinità che, secondo la topica dell'encomio secentesco, sono evocate per gareggiare con lei sono Febo e Pallade, che chiaramente impallidiscono di fronte alla Madama Reale. 3

All'encomio per la dedicataria seguono le ottave dell' «Auttora sotto nome di Artigemma» donate ad un gruppo di donne, riunite ad Albano nella villa della nobildonna romana Lavinia Buratti per la vendemmia, occasione ricca di pregnanza mitica, che, infatti, viene utilizzata da Artigemma per la costruzione di questo virtuoso gineceo di baccanti. La cornice mitica iniziale è offerta dal nome dell'ospite, che coincide con quello della principessa, figlia di Latino, che secondo il mito avrebbe sposato Enea, cui avrebbe dato un figlio, quel Silvio da cui discenderanno i re latini per mezzo dei quali il mito di Enea si connette, sublimandolo, a quello di Romolo e Remo. Se la mitica Lavinia «un Regno hebbe in dote», la «novella» Lavinia è «d'un core / ricca, ch'a mille Regni, e mille Fregi / la fama adorna» (ibidem, 22) e le ottave si concludono, come era accaduto nell'encomio di dedica alla Madama Reale, con la vittoria di Lavinia Buratti sulla principessa del mito, le cui virtù, straordinarie, finiscono per impallidire di fronte a questa «nuova Reina».

Portia Mazzarini, destinataria della seconda selva di ottave, è in realtà la seconda moglie di Pietro Mazzarino, padre del celebre cardinale e primo ministro francese Giulio Mazzarino, che era però nato dal primo matrimonio di Pietro, con la virtuosa Ortensia Bufalini. Le ottave costiane legano Porzia al cardinale francese, facendola assurgere al ruolo di madre.

Le selve successive sono per Chiara Felice, Contessa di Ripa, e per la Marchesa Giulia Malvezzi; in entrambi i casi la lode delle donne è connessa per paronomasia ai loro nomi: Chiara è la «ripa» cui si approda felici dopo la burrasca e Giulia non patisce, per le sue doti straordinarie, i «mal' vezzi» della fortuna avversa. Nel gruppo delle novelle baccanti le due nobildonne sono esclusivamente esaltate per la loro bellezza, che, ovviamente, è speculum animi.

Diversa intonazione e funzione hanno, invece, le ottave per Prudenza Buratti Massimi, che è incinta e, dunque, dovrà celebrare in sé il possesso di doti diverse:

Tra groppi d'oro con be' l'arte splende

Prudenza in tè di vaga stella immago 
Ed' ai raggi del Sol lieta s'accende.

Ad' annuntiar nel tuo bel seno il Tago

Di Castore, e Polluce in tè discende

Per la stella Oriental Parto presago

E di Lavinia in nobil fè ristretta

Romulo, e Remulo il Campidoglio aspetta.

$[\ldots]$

Tessi de i Giuli, e Massimi Corona

Ne la tua Prole vigorosa, e forte

Vaga Latina e già la fama suona

Ceder à l'opre lor vinta la sorte

Gode al tuo fertil sen lieta Pomona

Ed apre à te de suoi tesor le Porte

Lo Dio pennato di contenti Amante

Ed applaude à tuoi parti il gran Tonante.

(Ibidem, 33-34)

Le divinità dell'Olimpo sono scomodate per elogiare la donna incinta e la prole che da lei nascerà, ma neanche in questo caso i versi di Margherita Costa riescono ad affrancarsi dal lezioso tono di una civile conversazione tra donne, che, nel Seicento - dopo Il merito delle donne 4 di Moderata Fonte, 5 il celebre dialogo in cui sette donne veneziane tra loro amiche (una donna sposata da tempo, una sposa novella, una vedova giovane ed una anziana, una madre anziana, una figlia, un'intellettuale nubile) s'incontravano in una «casa bellissima», con un «giardino bellissimo» e, nel corso di due giornate, discutevano della condizione della donna e dei rapporti delle donne con gli uomini - è già diventata un tòpos da replicare. Di quella civile querelle i dodici componimenti di Artigemma per le nobildonne baccanti non sanno, purtroppo, che imitare la cornice. Anche la consolatio per Giulia Errera, rimasta vedova, non conosce sincerità d'intonazione, ma maniera, quella stessa che ritroviamo nell'elogio della bambina Lalla, figlia della padrona di casa.

Maggiore sincerità ed ispirazione sono invece rinvenibili nei componimenti successivi, il primo dei quali, dedicato Ad amante che parte dalla città mentre piove, preannuncia nel titolo un coinvolgimento emotivo che nei versi si tradurrà in una rappresentazione idillica dell'abbandono: 
Il Cielo è fatto oscuro, e senza luce,

il dì trà nembi di terror si mostra;

Febo d'impure macchie il volto inostra

E squalida l'Aurora il corso adduce.

Vacillante la terra à noi produce

Pallidi fiori, ed à la luce nostra

Fà d'atra notte formidabil mostra, che il Dio dè lo splendore altrove luce.

E l'Aria co'l suo pianto il pianto mio

Grave accompagna, ed à le mie procelle

Fulmini scote il fulminante Dio.

Piange il Ciel, piange il Sol, piangon le Stelle

A miei martiri; è per mio duol più rio

Trà i pianti, il Dio d'Amor vibra facelle.

(Ibidem, 50)

La natura, come in un classico idillio drammatico, si tinge dei colori più consoni allo stato d'animo dell'amante abbandonata. Per quanto il pianto del Cielo e della natura tutta ricordi atmosfere petrarchesche, i versi di Costa non conoscono i pentimenti del Canzoniere, né dubbi di carattere morale: il sonetto è chiuso, infatti, dall'immagine di Cupido che «vibra facelle», senza conoscere pietà, ma infierendo ulteriormente sull'amante abbandonata e accendendo in lei nuove fiaccole d'amore. La cura è autoctona e i sonetti della settimana sono la medicina che dovrà consolare, quando non guarire, le pene d'amore della donna abbandonata.

La Domenica, dies soli, è di Febo, il dio della poesia che guida il carro del Sole, a cui l'Autora deve l' «ardito stil» dei sonetti successivi, ognuno dei quali è dedicato ad una divinità connessa al giorno della settimana. Il lunedì è di Cinthia, uno dei nomina di Diana, personificazione della Luna; il martedì è di Marte, il dio che fa guerra, per antonomasia, ad Amore; il mercoledì è del dio che «spande d'eloquenza i detti» (ibidem, 54), Mercurio; il giovedì è del «Dio fulminante», Giove; il Venerdì è di Venere, «perfida» perché la luce dell'amante partito risplende ora «ad altri rai»; il sabato è di Saturno, il dio vecchio, «carco di cure» e nemico dell'amore che ama invece i giovani.

Il tema dell'abbandono, dell'amore tradito, torna anche nel sonetto che segue la serie della settimana. L'amante ha donato a Margherita un'ape d'oro, simbolo di fedeltà, ma le è stato infedele. Il sonetto gioca dunque sulla paronomasia 
«miele/fiele» per sottolineare la polivalenza dell'oggetto, ma anche l'instabilità delle passioni umane e la natura ingannevole d'amore.

L'impianto mitologico costituisce la struttura anche del sonetto successivo a quelli di tema amoroso, composto per una mostra d'armi a Testaccio, che diventa il teatro degli scontri tra Giove tonante e il bellicoso Marte, per raccontare di una pioggia improvvisa che costringe ad interrompere la manifestazione ecclesiastica.

A confermare il carattere rapsodico della Selva, s’incontra subito dopo un sonetto che ricalca uno dei temi più frequentati da Margherita Costa. L'Autora, infatti, in quasi tutte le sue opere, deve difendersi dall'accusa di non essere autrice dei suoi versi:

Non è for di ragione il titubare, ch'in Donna sia Virtude ai tempi d'oggi ove in ogn'alma par' che l'ozio alloggi, e il vitio in ogni sen goda imperare.

O' stelle questo secolo vulgare $\mathrm{Fa}$, che lunge da noi virtù sen poggi: e l'ignoranza in vece sua s'appoggi ove gran nobiltà pompe à più chiare.

Ma non però, che l'ignoranz'annida deve qual lei stimar mia penna frale, e de la fama mia farsi omicida.

Margherite ama Febo, ed è fatale Che con rai di virtude ei mi sia guida, mentre fra l'ombre giace ogni mortale.

(Ibidem, 60)

L'incipit del sonetto rivela che l'Autora è ben consapevole della generale reticenza del mondo delle lettere nei riguardi delle donne, a cui poco ritiene si addica la poesia, soprattutto se amorosa e licenziosa. Anche in una delle sue liriche, inserita nella raccolta di Componimenti poetici delle più illustri rimatrici d'ogni secolo che Luisa Bergalli pubblicò nel 1726 in due parti (BERGALli 1726, 151-52), la Costa chiosava così un luogo autobiografico del testo:

Canterò dunque senza aver paura, 
che il mondo, il Ciel, o i Fati rei facciano contro me mala congiura.

Ma so, che vi saranno più di sei, e le dozzine intere che diranno, che se i versi son buoni non son miei.

(CosTA 1638, 4-5)

È interessante, credo, notare che in entrambi i casi l'autrice suggerisce non tanto una sua presunta superiorità, quanto una preclusione del mondo letterario maschile nei riguardi delle donne che fanno poesia. $\mathrm{E}$ forse, in questo libro, disordinato come una selva di erbe diverse e d'animali, non è un caso che a seguire il sonetto ve ne siano due dedicati alla compassione per una donna che in Chiesa piange le sue pene d'amore, chiedendo perdono per il solo fatto d'aver osato amare con i sensi, come un uomo.

Il sonetto successivo, Argomenti morali, infatti, sembra raccontare i pensieri della donna penitente che riflette sulla vanità del tutto quando non s'ama riamati:

Momentaneo è il gioire, eterno il duolo, fallaci le grandezze, e i pensier vani, ed'ogni bene sé né fugge à volo.

(COSTA 1647, 63)

Sono ancora donne che amano anche le protagoniste dei sonetti successivi. L'amore narrato è sempre sofferto: lui tradisce, lui è incurante dell'amore di lei, è lontano, riparte senza preavviso, è ingrato, è malinconico. A soffrire per amore è sempre la donna, perché è una donna che resta, mentre l'uomo continua ad andare. L'abbandono è visto, nei versi della Costa, come un atto di tirannia crudele, l'ennesima affermazione della libertà dell'uomo, della sua autonomia completa rispetto alla donna. Alla donna resta la poesia, attraverso la quale dar voce ad un male che affligge l'io in molte opere di Margherita Costa: la gelosia, alla quale nella Selva sono destinati due sonetti consecutivi, indirizzati al medesimo amante.

Con ali nere, e crin di serpi avvinto 
mostro c'hà man di gel mi stringe il core:

e con tremanti brine il caro ardore

sparge di sangue, e di timore tinto.

Resta lo spirto nel dolor convinto,

l'anima dentro il sen rinasce, e more:

e sparso il volto di mortal pallore

sembra trà vivi già di vita estinto.

Ah perfido, e perche d'altra nel seno

godi, e mercé d'Amore à mè non dai

e spargi il mio gioir d'atro veneno!

Tu mi dicesti, che mentir non sai

e pur'è volto, in ombra il mio sereno,

mà chi puotè ingannar non amò mai.

(Ibidem, 71)

Il sonetto evidenzia la topica somatizzazione della gelosia, o dovremmo dir meglio, la fisiognomica della gelosia che, per prima, proprio una poetessa, l'antica Saffo, aveva tradotto in versi. Caldo e freddo, fuoco e ghiaccio, rossore e pallore, nero e bianco costituiscono la tavola cromatica di questo male, incurabile, innescato dal tradimento, dalla menzogna - «chi puoté ingannar non amò mai» - che nasce direttamente nell'anima e, dunque, logora lentamente lo «spirto» vitale della donna.

Sonetti cromatici sono anche i tre successivi, che una donna «vestita di nero» scrive all'amante infedele: l'abito del lutto, che fa risaltare il colore bianco della pelle pura di lei, indica la morte della fedeltà, non certo dell'amore che continua ad accender fuochi rossi e incandescenti.

I successivi sonetti ripetono il motivo dell'amante abbandonata, dimenticata, trascurata, tradita. La donna subisce l'amore e i suoi capricci, ma soprattutto, pur senza conoscere i turbamenti dell'animo dimidiato tra terra e Cielo del maestro Petrarca, la donna che sceglie l'amore di un uomo perde quello di Dio, perché sceglie la passione fisica, accetta la prostrazione, l'inevitabile sottomissione ad un amante padrone, che ha su di lei quasi potere di vita o di morte, dal momento che per Margherita Costa vivere senza amare è come esser morti.

La Selva di Diana si chiude con due donne: la prima è Anna d'Asburgo, regina di Francia quando Margherita Costa arriva a Parigi, e la seconda è l'Autora, che chiosa il libro con una selva di ottave autobiografiche. L'explicit 
conferma, a mio giudizio, il carattere composito dell'opera e la mancanza di un progetto ecdotico. Se, infatti, il libro si fosse chiuso con Per gl'honori ricevuti in Parigi dalla Maestà della Regina di Francia si sarebbe potuta ipotizzare una coerenza interna, una progettualità narrativa: La Selva di Diana si apriva con Maria Cristina di Savoia, sorella di Luigi XIII, e si chiudeva con sua moglie, configurandosi come la lode del passato e del futuro del re che l'aveva accolta presso la sua grandiosa corte. Ma la Partenza di Roma de l'Autora del anno 1647 posta ad explicit del libro pregiudica questa possibilità esegetica. Quest'ultima selva di ottave è interamente concepita come una lamentatio. La terra che le ha dato i natali e che dovrebbe essere per lei patria ha reso la poetessa «povera d’ogni ben e, inerme, e nuda / di speme» (CoSTA 1647, 90); ma se il «piè» parte, il «cor» stenta a lasciare Quirino, nonostante il suolo patrio sia stato avaro d'onori. Il tòpos dei seguaci di Febo, come la Costa proclama più volte di essere in queste ottave, ricorda in modo vivido i luoghi autobiografici della Lira mariniana dell'Adone; anche là il poeta lamentava d'essere stato respinto e torturato proprio dalla «Sirena disleal» che gli aveva dato i natali (MARINO 1602, 209). Come il poeta barocco per eccellenza, che resta modello evidente di buona parte della sua produzione, Margherita Costa tiene a chiosare la Selva di Diana con questa sfraghis della poetessa esule suo malgrado, che lascia che «il volgo rio» di Roma, quella stessa città che aveva cacciato qualche decennio prima la pittora Artemisia Gentileschi, «ad altri mova aspra tenzone» (Costa 1647, 95). «A novo Ciel» la poetessa - cui la città di Quirino ha calpestato «superba» il «lauro suo» - parte, ad inseguir miglior destino, lei che è stata «sempre tra scherni e tra miserie involta»:

Nata per intrecciar speranze ignote, cui solo è dato sormontare il volo sovra le sfere per cader nel suolo.

(Ibidem, 86) 


\section{Note}

${ }^{1} \mathrm{Su}$ Margherita Costa (Roma, 1600 ca.- 1657 ca.) pochi sono i contributi critici rilevanti: Bianchi 1924-1925; Capucci 1984; De Liso 2019 e 2020; Di Maro 2020; Ferrone 1986; Morandini 2001, 114-124, 238; Costa-Zalessow 2010 e 2015; Piantoni 2017 e 2018, Robarts 2019.

${ }^{2}$ L'indice del libro è il seguente: All'Altezza reale di Madama Savoia; Ai Lettori; L'Alpi; L'Auttora sotto nome di Artigemma; Alla Illustrissima Signora Portia Mazzarini; All'Illustrissima Signora Chiara Felice Contessa Ripa; Alla Illustrissima Signora Marchesa Giulia Malvezzi; Alla Illustrissima Signora Prudenza Buratti De Massimi, All'Illustrissima Signora Ottavia Vitelleschi; All'Illustrissima Signora Porzia Crivelli; Alla Illustrissima Signora Giulia Errera Vedova; Alla Signora Eustochia Panizza; Alla Signora Caterina Lopes; Alla Illustrissima Signora Donna Girolama Malvezzi; Alla Signora Lalla Bambina, figlia de la Signora Lavinia Buratti; All'Illustrissima Signora Maria Vica alludendo al suo nome; Ad amante che parte dalla città mentre piove; Al Medesimo Passione amorosa per ciaschedun giorno della settimana. Domenica, Lunedi, Martedi, Mercordi, Giovedi, Venerdi, Sabbato; Ad amante infedele per un'ape d'oro donata da lui; Nel mese di Luglio fu interrotta da'i fulmini la mostra dell'Armi ecclesiastiche nella pianura del Monte Testaccio; A persona che dice esservi chi teme che l'Autora non operi da sé nelle sue compositioni; A Bella donna in atto di Penitenza avanti il Confessore; Alla medesima mentre scalza va' premendo il suolo in atto di devotione; Argomenti morali, donna cinta d'un cordone al suo Amante; Di lontananza; Donna di nome Margherita ad amante, ch'appena ritorna dalla campagna, che di novo si parte per quella; Rimprovero d'ingratitudine al suo Amante; Nel vedere il suo Amante malinconico; Ad amante, che con atti di crudeltà vanta d'esser un altro Dionigi in tiranneggiar l'amata; Al medesimo; Di Gelosia; Al medesimo; Amante vestita di nero ad amante infedele; Al medesimo mentre la prega vestirsi di nero; Al medesimo d'Infedeltà; Ad amante temerario; Bella donna ad Amore mentre è avanti il Confessore; Passione amorosa ad amante crudele; Per gl'honori ricevuti in Parigi dalla Maestà della Regina di Francia; Partenza di Roma de l'autora del anno 1647.

${ }^{3} \mathrm{Cfr}$. «Lo schema di siffatti encomi non varia: affermato che un principe o una principessa possiede una certa dote, e dimostrata con esempi la veridicità dell'asserto, si passa a dichiarar come nessuno dei grandi personaggi della mitologia, dei tempi antichi e dei recenti, andato famoso per la medesima facoltà, sia comparabile al moderno magnate. E se si tratterà di principesse, investite della somma autorità, come avviene in Francia e in Piemonte, si canterà, oltre che la bellezza, l'animo virile e la mente profonda, che ne raccomandan il nome ai posteri» (Bianchi 1924-1925, 175-76).

${ }^{4}$ Il Merito delle Donne scritto da Moderata Fonte in due giornate. Ove chiaramente si scuopre quanto siano elle degne e più perfette de gli huomini fu pubblicato a Venezia, presso Domenico Imberti, nel 1600. L'opera è stata edita per la cura di Adriana Chemello (Chemello 1988).

${ }^{5}$ Su Moderata Fonte si vedano almeno: Malpezzi Price 2003; Martelli 2011; Pezzini 2012. 


\section{Bibliografia}

BARBERI SQUAROTTI 2018:

Il mito di Diana nella cultura delle corti. Arte Letteratura Musica, a c. di Giovanni Barberi Squarotti, Annarita Colturato e Clara Goria, Firenze, Olschki, 2018.

BERGALLI 1726:

Luisa BERGALLI, Componimenti poetici delle più illustri rimatrici d'ogni secolo, Venezia, Appresso Antonio Mora, 1726, vol. II, pp. 149-54.

BIANCHI 1924-1925:

Dante BIANCHI, Una cortigiana rimatrice del Seicento: Margherita Costa, in «Rassegna critica della letteratura italiana», XXIX (1924), pp. 187-203 e XXX (1925), pp. 158-211.

CAPUCCI 1984:

Martino CAPUCCI, Costa, Margherita, in Dizionario biografico degli italiani, Roma, Enciclopedia Treccani, 1984, vol. 30, pp. 232-34.

\section{CASTELNUOVO 2007:}

La reggia di Venaria e i Savoia. Arte, magnificenza e storia di una corte europea, a c. di Enrico Castelnuovo et alii, catalogo della mostra, Torino, Allemandi, 2007, 2 voll.

CHEMELLO 1988:

Moderata Fonte, Il Merito delle Donne scritto da Moderata Fonte in due giornate. Ove chiaramente si scuopre quanto siano elle degne e più perfette de gli huomini, a c. di Adriana Chemello, Venezia, Eidos, 1988.

COSTA 1638:

Margherita CosTA, La Chitarra, Francoforte, Daniel Watsch, 1638.

COSTA 1647:

Margherita Costa, La Selva di Diana. Opera di Margherita Costa Romana, dedicata all'Altezza Reale di Madama di Savoia, A Parigi, per Sebastiano Cramoisy, stampatore ordinario del Re, e della Regina Regente, 1647.

COSTA-ZALESSOW 2010:

Natalia COSTA-ZALESSOW, Una poesia femminista del 1672 anonima e dimenticata, da attribuire a Margherita Costa, in «Esperienze letterarie», 35 (2010), 4, pp. 7985 .

COSTA-ZALESSOW 2015:

Natalia Costa-ZALEssow, Voice of a Virtuosa and Courtesan. Selected poems of Margherita Costa, New York, Bordighera Press, 2015.

DE LISO 2019a:

Daniela DE LISO, Tra le scritture femminili del Seicento: prime indagini, in «Tutto ti serva di libro». Studi di Letteratura italiana per Pasquale Guaragnella, Lecce, Argo, 2019, vol. I, pp. 460-74.

DE LISO 2019b:

Daniela DE LISO, Veronica Franco, poetessa meretrice per un Umanesimo al femminile, in La Aportaciòn de la mujer en la Construcciòn, Deconstrucciòn y 
Redefiniciòn del Humanismo, a c. di Vicente Gonzàlez Martin, Celia Aramburu Sanchez, Nicola Florio y Giulia Di Santo, Salamanca, Edicion Universidad Salamanca, 2019, pp. 35-48.

DE LISO 2020:

Daniela DE LISO, Le lettere amorose di Margherita Costa, in (Auto)narrativas: hacia la construcción de un canon alternativo en italiano, a c. di Sara Velázquez García e Laureano Núñez García, Salamanca, Ediciones Universidad de Salamanca, 2020, pp. 47-64.

DI MARO 2020:

Maria DI MARO, Una poetessa del XVII secolo: Margherita Costa, in (Auto)narrativas: hacia la construcción de un canon alternativo en italiano, a c. di Sara Velázquez García e Laureano Núñez García Salamanca, Ediciones Universidad de Salamanca, 2020, pp. 81-97.

FERRONE 1986:

Siro FERRONE, Nota biobibliografica, in Commedie dell'arte, Milano, Mursia, 1986, 2 voll., II, pp. 235-38.

GRISERI 1988:

Andreina GrISERI, Il Diamante. La Villa di Madama Reale Cristina di Francia, Torino, Istituto Bancario San Paolo, 1988, pp. 195-96.

MALPEZZI PRICE 2003:

Paola MALPEZZI PRICE, Moderata Fonte: women and life in sixteenth-century Venice, London, University Press, 2003.

MARINO 1602:

Giovan Battista MARINO, Rime, Venezia, Ciotti, 1602.

MARTELLI 2011:

Daria MARTELLI, Polifonie: le donne a Venezia nell'età di Moderata Fonte, pref. di Federica Ambrosini, Padova, CLEUP, 2011.

MORANDINI 2001:

Giuliana MoRANDInI, Sospiri e palpiti. Scrittrici italiane del Seicento, Genova, Marietti, 2001.

ODORISIO 1979:

Ginevra Conti Odorisio, Donna e società nel Seicento, Firenze, Hoepli, 1979.

PEZZINI 2012:

Serena PEZZINI, Il merito delle donne, Dialogo di Moderata Fonte. Prove generali di un futuro impossibile, in Futuro italiano. Scritture del tempo a venire, a c. di Alessandro Benassi, Fabrizio Bondi, Serena Pezzini, Pisa, Pacini Fazzi, 2012, pp. 144-58.

\section{PIANTONI 2017:}

Luca PiANTONI, L'epistolario amoroso di Margherita Costa, in L'Italianistica oggi: ricerca e didattica, Atti del XIX Congresso dell'ADI - Associazione degli Italianisti (Roma, 9-12 settembre 2015), a c. di Beatrice Alfonzetti, Teresa Cancro, Valeria Di Iasio, Ester Pietrobon, Roma, Adi editore, 2017, pp. 1-5. 
PIANTONI 2018:

Luca PIANTONI, Le lettere amorose di Margherita Costa tra sperimentalismo e 'divertissement', in «Studi Secenteschi», 59 (2018), pp. 33-52.

ROBARTS 2019:

Julie L. RoBARTS, Challenging Male Authored Poetry: Margherita Costa's Marinist Lyrics (1638-1639), Tesi di dottorato, The University of Melbourne, Australia, 2019.

VARALLO 2010:

Franca VARALlo, Il tema della caccia nelle feste sabaude nei secoli XVI e XVII, in La caccia nello stato sabaudo, a c. di Paola Bianchi a Pietro Passerin d'Entrèves, Torino, Zamorani, 2010, vol. 1, pp. 137-44. 